\title{
A probabilistic multi-objective approach for FACTS devices allocation with different levels of wind penetration under uncertainties and load correlation
}

\author{
M. EL-Azab ${ }^{1}$, W. A. Omran², S. F. Mekhamer ${ }^{3}$, H. E. A. Talaat ${ }^{4}$ \\ ${ }^{1}$ Electrical Engineering Department, Ain Shams University, Egypt \\ ${ }^{2,3,4}$ Electrical Engineering Department, Future University, Egypt
}

Article Info
Article history:
Received Apr 3, 2019
Revised Jan 16, 2020
Accepted Feb 26, 2020

Keywords:

FACTS

MOPSO

Multi-objective optimization

Probabilistic load flow

Two points estimation method

Wind energy

\begin{abstract}
This study presents a probabilistic multi-objective optimization approach to obtain the optimal locations and sizes of static var compensator (SVC) and thyristor-controlled series capacitor (TCSC) in a power transmission network with large level of wind generation. In this study, the uncertainties of the wind power generation and correlated load demand are considered. The uncertainties are modeled in this work using the points estimation method (PEM). The optimization problem is solved using the multi-objective particle swarm optimization (MOPSO) algorithm to find the best position and rating of the flexible AC transmission system (FACTS) devices. The objective of the problem is to maximize the system loadability while minimizing the power losses and FACTS devices installation cost. Additionally, a technique based on fuzzy decision-making approach is employed to extract one of the Pareto optimal solutions as the best compromise one. The proposed approach is applied on the modified IEEE 30-bus system. The numerical results evince the effectiveness of the proposed approach and shows the economic benefits that can be achieved when considering the FACTS controller.
\end{abstract}

Copyright (C) 2020 Institute of Advanced Engineering and Science. All rights reserved.

\section{Corresponding Author:}

M. EL-Azab,

Electrical Engineering Department,

Ain Shams University,

Cairo-Egypt.

Email:mahrous@osman.net

\section{INTRODUCTION}

In recent years, the current growth in demand for electricity and rising population of the world are forcing electric utilities to take benefit of renewable energy sources (RESs) in the generation mix. In recent years, the interest in RESs is more and more increasing, mainly because of the rising concern to environmental issues and of the decreasing investment cost for such systems, also thanks to very favorable national policies of financial support. One of the greatest widely used renewable energy source is the wind, where the global installed capacity of wind energy systems has reached 591GW in 2018 [1]. Moreover, the fluctuations in the wind power causes vulnerabilities to the power system and prompts technical challenges on the network operation [2].

The application of F'ACTS controllers, which are based on power electronic switches, in power systems has been increasing [3]. These controllers supply reactive power compensation, which can increase the maximum transfer capability of electrical network, and therefore, can be utilized to enhancement the different performance parameters of the electric network in dynamic state and steady state [4]. The efficiency of the FACTS devices in improving the network performance mostly depend on their size and location. In the scientific publications, defining the ideal locations and compensation degree of FACTS 
controller have been deliberated widely. Several approaches have been intended to settle the FACTS allocation issue.

The methods used for the allocation of FACTS controller can be split into three basic groups; sensitivity based indices [5], classical optimization based and metaheuristic methods [6]. We can see from the literature review from last decade that has focused on several rule have been deliberated in allocation issue. An a portion of the expressed goals in the literature are : network loadability enhancement, line thermal constraints violation [7]. Some interesting studies are focused on the investigation of loss reduction, voltage profile enhancement $[8,9]$, voltage stability improvement, fuel cost reduction [10] ,reliving transmission line congestion [11] and enhancement available transfer capability (ATC) [12]. Each of the above stated objectives enhance power system performance. Be that as it may, improvement in one goal doesn't guarantee a similar upgrade in others. Along these lines, none of the expressed specialized goals can't be surrendered in the allocation of FACTS controller. Therefore, the allocation of FACTS controller due to one or more objectives while not taking into thought the cost of the devices is not a practical one. In detail, the optimal allocation of FACTS controller should be expressed as multi-objective optimization (MOP) issue from both economical and technical points of views. Furthermore, most of the present research are established on a supposition that there is no variation in the demand load over time. These approaches have been carried out under a fixed load profile or overloaded conditions.

Considering uncertainty of the input parameters of the power system, various methods have been planned for estimating the state of the power system. The most perfect technique is Monte Carlo simulation (MCS), which is usually used as standard method [13]. MCS provides the most proper outcomes but the calculation is much time- exhaustion; thus, it is not appropriate for real time implementation. So as to diminish the computational exertion related with simulation-based strategies, analytical and estimation techniques have been utilized. Approximation methods consist of PEM [14]. Cumulant method (CM) is presently the illustrative of analytical procedures [15]. In [16] a probabilistic techniques for the sizing of multiple FACTS controller in power systems for steady-state voltage profile improvement using MCS technique are used. In [17] also, optimal allocation of FACTS controllers to minimize the cost of generation was solved by differential evolution algorithm DE in coincidence with Monte Carlo simulation (DE-MCS) taking into account uncertainty in load and wind generation output. In [18] a multi-objective function was proposed for optimal allocation of a UPFC in power system considered the maximization of system predictability and minimization active power loss using NSGA-II. However, these papers have not paid attention to and the load correlation on power system.

This paper presents an approach based on multi-objective functions for solving the allocation problem of FACTS devices considering high penetration level of wind energy under uncertainties in demand and wind generation output. Moreover, the load correlation is considered. The proposed algorithm MOPSO with combined 2PEM (MOPSO-PEM) is introduced for FACTS controller allocation problem under uncertainties. This paper proposes the use of 2PEM instead of MCS, because PEM is much faster than MCS in solving probability optimal power flow (POPF) problems. The reminder of the paper is organized as follows: modeling of the uncertainties is given in section 2. Mathematical modeling of FACTS is present in section 3. The 2PEM for PPF calculation is defined in section 4. The problem formulation is present in section 5. The proposed (MOPSO-2PEM) is described in section 6. In section 7, case study and the simulation results are presented. Conclusions are presented in section 8 .

\section{MODELING OF SYSTEM UNCERTAINTIES}

The system loads are modeled as PQ buses with definite active and reactive power and the wind farm output is modeled as negative load. Famously, the loads ought not to be considered as a deterministic value. Rather, they are generally demonstrated as a typical of Gaussian probability density function (PDF) whose mean is equivalent to an expected value. In utmost cases, the standard deviation of the PDF is a fraction of the estimated load value. In this study the load in each bus is demonstrated with mean $(\mu)$ equal to the base load and standard deviation $(\sigma)$ is assumed to be $\pm 7 \%$ of the base load. The normal distribution of the load demand (d) is presented as $[19,20]$ :

$$
\mathrm{F}(\mathrm{d})=\frac{1}{\sigma \sqrt{2 \pi}} * \exp \left(\frac{\left(-(\mathrm{d}-\mu)^{2}\right)}{2 \sigma^{2}}\right)
$$

where, the variation of wind power output is an uncertain parameter, which can be modeled using historical data records of the wind speed. In this work, variation of the wind speed, $v$, is modeled using Weibull PDF as follow [21]: 


$$
\operatorname{PDF}(v)=\left(\frac{k}{c}\right)\left(\frac{v}{c}\right)^{k-1} \exp \left[-\left(\frac{v}{c}\right)^{k}\right]
$$

where $k$ is shape parameter and $c$ : is scaling parameter $\mathrm{m} / \mathrm{s}$.

It is assumed that in each region, the PDF of wind speed is known, therefore, the transformation of wind speed to wind turbine output power is given by:

$$
P W=\left\{\begin{array}{cl}
0, & 0 \leq v \leq v_{i} \\
P_{r}\left(\frac{v-v_{i}}{v_{r}-v_{o}}\right), & v_{i} \leq v \leq v_{r} \\
P_{r}, & v_{r} \leq v \leq v_{o} \\
0, & v>v_{o}
\end{array}\right.
$$

where, $V_{i}, V_{o}$ is the cut-in and cut-out wind speed, $V_{r}$ is the rated speed and $\operatorname{Pr}$ is the rated power.

\section{FACTS DEVICES AND MODELING}

FACTS controller technology includes a set of controllers that provide control over one or more transmission systems parameters. These FACTS controller can coordinate with control units or operate stand-alone. FACTS controller use power electronics converters. These converters control the power flows and improve the usage of the transmission's lines. In literature, the current steady-state models of FACTS controller can be classified as two categories: power injection model and variable reactance modeling.

\subsection{SVC model}

SVC is used as a controlled source of the reactive power. The main feature of SVC is to provide voltage stability without using large reactors and banks of capacitors to absorb or supply reactive power. Thus, a SVC control its inductive or capacitive current independently of the system voltage. The SVC can consume or inject reactive power in the connected bus. If the bus voltage is lower than the required voltage, the SVC injects reactive power into this bus to increase its voltage; this case is similar to a capacitive behavior as shown in Figure 1 (a) However, when the bus voltage is above the required voltage, the SVC absorb reactive power from this bus to decrease its voltage; this case is similar to inductive behavior. The SVC bus in the network can be represented by absorbed or injected reactive power. The working range of is set -100 to 100 MVAR [22].

$$
\operatorname{MVAR}_{\mathrm{i}}^{\min } \leq \mathrm{MVAR}_{\mathrm{i}} \leq \mathrm{MVAR}_{\mathrm{i}}^{\max }
$$

\subsection{TCSC model}

TCSC acts as a series-controlled reactance, which aim to compensate the impedance of the transmission lines. The switching of the TCSC give a mechanism to control power flow, which allows rising the load of the existing grid. In addition, TCSC can damp the inter area oscillation of the large electrical network, and offers an opportunity of power flow adjustment in response to different contingencies that may take place in electrical network. Also, The TCSC can regulate the steady-state power flow to retain it within the line's thermal limits. The TCSC are considered as a variable reactance $\left(X_{T C S C}\right)$ which is connected in series with the transmission line as shown in Figure 1 (b). The variation of $X_{T C S C}$ provide regulating active power through the transmission line. TCSC is implemented using a controlled series reactor in parallel with a fixed series capacitor. Consequently, to guarantee the system stability, it is advocate to compensate up to compensating degree $\left(K_{s e}\right)$ of the line nominal reactance value $\left(\mathrm{X}_{\text {line }}\right)$, both in inductive $\left(K_{s e, \text { ind }}\right)$ and the capacitive $\left(K_{s e, \text { cap }}\right)$ operating zones. As a result, the total branch reactance $\left(\mathrm{X}_{\mathrm{ij}}\right)$ is as follows [23].

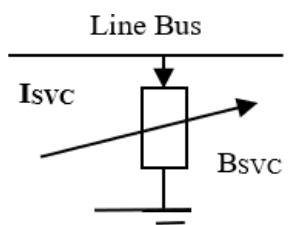

(a)

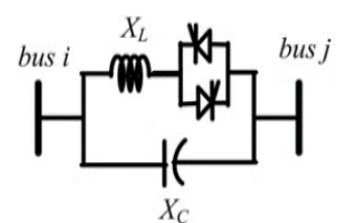

(b)

Figure 1. Models of FACTS controller (a) SVC (b) TCSC 
The equivalent reactance of branch is:

$$
\begin{aligned}
& X_{i j}=X_{\text {line }}+X_{T C S C} \\
& X_{T C S C}=K_{\text {Se }} X_{\text {line }}
\end{aligned}
$$

where, $K_{\text {se }}$ and $X_{\text {line }}$ are compensation degree and branch reactance, respectively. $X_{T C S C}$ is the TCSC reactance. The degree of the practical compensation of the TCSC varies between $80 \%$ capacitive and $20 \%$ inductive [23].

\section{PROBABLISTIC LOAD FLOW}

The deterministic power flow analysis of the performance of the system relies on certain specific scenario and ignore the uncertainty within the parameters of the system and the states. It considers that all the states are known and fixed. On the other hand, a probabilistic approach, evaluates the probability distribution for the uncertain variables, consequently, well reflect the definite system performance. Several methods for probabilistic load flow (PLF) study have been established. These methods fall in three basic groups: analytical methods, MCS procedures, and approximate methods. Point estimate method, PEM, is as of now the delegate of approximate methods for PLF calculations. In this study, the three-point estimate $(2 \mathrm{PEM}+1)$ method is applied here to handling the uncertainty effects [14, 24]. The original 2PEM cannot handle correlated uncertain variable. To solve the probabilistic optimal power flow with correlated variables, the covariance matrix transformation method [25] is combined into the original PEM method was used. In this study the correlation coefficient is set to 0.7 .

\section{1. $(2 \mathrm{~m}+1)$ algorithm}

Step 1: Determine the number of uncertain parameters $m$.

Step 2: Determine the locations of concentrations $\xi_{l, 1}, \xi_{1,2}, \xi_{l, 3}$ and the probabilities of concentrations $W_{l, 1}$, $W_{l, 2}$ and $W_{l, 3}$ for each variable $l$.

$$
\begin{aligned}
& \xi_{l, 1}=\frac{\lambda_{l, 3}}{2}+\sqrt{\lambda_{i, 4}+3 *\left(\frac{\lambda_{1,3}}{2}\right)^{2}} \\
& \xi_{1,2}=\frac{\lambda_{1,3}}{2}-\sqrt{\lambda_{i, 4}+3 *\left(\frac{\lambda_{1,3}}{2}\right)^{2}} \\
& \xi_{l, 3}=0
\end{aligned}
$$

where $\lambda_{l, 3}$ and $\lambda_{l, 4}$ mean the skewness and kurtosis of the random input parameter $l_{i}$.

$$
\begin{aligned}
& \lambda_{\mathrm{i}, 3}=\frac{\int_{-\infty}^{\infty}\left(\mathrm{l}_{\mathrm{i}}-\mu_{\mathrm{i}}\right)^{3} \mathrm{f}_{\mathrm{i}} \mathrm{dx} \mathrm{x}_{\mathrm{i}}}{\sigma_{\mathrm{i}}{ }^{3}} \\
& \lambda_{\mathrm{i}, 4}=\frac{\int_{-\infty}^{\infty}\left(\mathrm{l}_{\mathrm{i}}-\mu_{\mathrm{i}}\right)^{4} \mathrm{f}_{\mathrm{i}} \mathrm{dx_{ \textrm {i } }}}{\sigma_{\mathrm{i}}{ }^{4}} \\
& \mathrm{w}_{\mathrm{l}, \mathrm{k}}=(-1)^{3-k} / \xi_{i, k}\left(\xi_{l, 1}-\xi_{l, 2}\right), \mathrm{k}=1,2 \\
& w_{l .3}=\frac{1}{m}-\frac{1}{\left(\lambda_{l, 4}-\lambda_{l, 3}^{2}\right)}
\end{aligned}
$$

Step 3: Determine the three concentrations $\mathrm{p}_{\mathrm{l}, \mathrm{k}}, \mathrm{k}=1: 3$ for each parameter $l$.

$$
\begin{aligned}
& \mathrm{p}_{l, 1=} \mu_{\mathrm{pl}}+\xi_{\mathrm{l}, 1} \sigma_{\mathrm{pl}} \\
& \mathrm{p}_{l, 2=\mu_{\mathrm{pl}}}+\xi_{\mathrm{l}, 2} \sigma_{\mathrm{pl}} \\
& \mathrm{p}_{\mathrm{l}, 3=\mu_{\mathrm{pl}}}
\end{aligned}
$$

where $\sigma_{\mathrm{pl}}$ and $\mu_{\mathrm{pl}}$ are standard deviation and the mean respectively. 
Step 4: calculate the objective output $f_{l, k}$ for all concentrations using DOPF.

$$
f_{l, k}(X, u)=f_{l, k}(X, u)\left(\left[\mu_{p 1}, \mu_{p 1}, \mu_{p 1}, \ldots, p_{l, k}, \ldots, \mu_{p 1}\right], \mu\right)
$$

where, $\quad k=1: 3, \quad l=1: m, x$ is the concentration $\left[\mu_{\mathrm{p} 1}, \mu_{\mathrm{p} 1}, \mu_{\mathrm{p} 1}, \ldots \ldots, \mathrm{p}_{\mathrm{l}, \mathrm{k}}, \ldots, \mu_{\mathrm{p} 1}\right]$ and $u$ is the vector of control variables.

Step 5: calculate $E(Y)$ and $E\left(Y^{2}\right)$.

$$
\begin{aligned}
& \mathrm{E}(\mathrm{Y})=\sum_{l=1}^{\mathrm{m}} \sum_{\mathrm{k}=1}^{3}\left(\mathrm{w}_{\mathrm{l}, \mathrm{k}} * f_{l, k}(\mathrm{X}, \mathrm{u})\right. \\
& \mathrm{E}(\mathrm{Y})^{2}=\sum_{l=1}^{\mathrm{m}} \sum_{\mathrm{k}=1}^{3}\left(\mathrm{w}_{\mathrm{l}, \mathrm{k}} * f_{l, k}(\mathrm{X}, \mathrm{u})^{2}\right)
\end{aligned}
$$

Step 6: Calculate the expectation and standard deviation for the objective output using:

$$
\begin{aligned}
& \mu=E(Y) \\
& \sigma=\sqrt{E\left(Y^{2}\right)-(\mu)^{2}}
\end{aligned}
$$

\section{PROBLEM FORMULATION AND OBJECTIVES FUNCTION}

\subsection{Problem statement}

The goal in this study is to improve the electrical network loadability considering static security in terms of line loading and voltage level through optimal allocation of FACTS controller. Two categories of FACTS controller, namely TCSCs and SVCs are located in order alleviate the bus voltage and overloads violations. The OPF problem considering the presence of TCSC and SVC. The MOP can be mathematically defined as [26]. Minimize $\mathrm{x}$ in,

$$
\begin{aligned}
& F(x)=\left\{F_{1}(x), F_{2}(x), \ldots \ldots F_{N F}(x)\right\} \\
& \text { Subject to }\left\{\begin{array}{l}
g_{1}(x), g_{2}(x), \ldots \ldots g_{N G}(x)=0 \\
h_{1}(x), h_{2}(x), \ldots \ldots, h_{N H}(x) \leq 0
\end{array}\right.
\end{aligned}
$$

where $N F$, is the number of objective functions, $N G$ is the number of inequality constraints and $N H$ is the number of equality constraints.

In the MOP, more than one objective function is being optimized simultaneously. Most of the time, one common solution could not be found for all the objective functions in the search space. In contrast, a set of best points could be achieved. This set of points is referred as the Pareto solution set. The values of the Pareto optimal points in the objective space form Pareto front in the objective space. The solution for this kind of problems requires points in the objective space should converge to the Pareto front and cover the front [27].

\subsection{Objective functions}

In this study, the objective functions considered the maximizing system loadability within system security constraint, minimizing the expectation of the real power losses in the transmission lines, and minimizing the establishment cost of FACTS controller.

\subsubsection{Minimization of real power losses $P L$}

The objective is to minimize the active power loss in the transmission network can be formulated as:

$$
f_{1}(\mathrm{x}, \mathrm{u})=\sum_{k=1}^{n l} g_{k}\left[\mathrm{~V}_{\mathrm{i}}^{2}+\mathrm{V}_{\mathrm{j}}^{2}-2 \mathrm{~V}_{\mathrm{i}} \mathrm{V}_{\mathrm{j}} \cos \left(\delta_{\mathrm{i}}-\delta_{j}\right)\right]
$$

where $g_{k}$ and $n l$, is the conductance of branch $k$, and the number of branches respectively. 


\subsubsection{Maximization of the system loadability}

The loadability of electrical network is the ability for swelling as much as conceivable the power transmitted by the network to the customers, and keeping the electrical network in a safe state in terms of branch loading and voltage levels.

$$
\text { Maximize }\left\{\lambda_{f}\right\}
$$

The load factor $\lambda_{f}$, is defined as follows:

$$
\begin{aligned}
& E\left[P_{D i}\left(\lambda_{f}\right)\right]=\lambda_{f} *\left[E\left(P_{D i}\right)\right] \\
& E\left[Q_{D i}\left(\lambda_{f}\right)\right]=\lambda_{f} *\left[E\left(Q_{D i}\right)\right] \\
& \lambda_{f} \in\left[1, \lambda_{f}{ }^{m a x}\right]
\end{aligned}
$$

where $P_{D i}, Q_{D i}$ are the active and reactive load demand at load bus $i$ respectively.

The objective function is based on indexes calculating the system loadability is expressed as:

$$
f_{2}(x, u)=\frac{1}{\lambda_{f}}
$$

\subsubsection{FACTS installation cost}

Another objective function to be considered, is minimizing the FACTS controller establishment cost that can be calculated as follows:

$$
f_{3}(x, u)=1000 * C * S
$$

where $C$ is the establishment cost of FACTS controller in US\$ /KVAR and Where $S$ is the installed value of the FACTS controller in MVAr. The cost of TCSC and SVC can be calculated according to the following [7]:

$$
\begin{gathered}
C_{S V C}=0.0003 S^{2}-0.3051 S+127.38 \mathrm{US} \$ / \mathrm{KVAr} \\
C_{T C S C}=0.0015 S^{2}-0.7130 S+153.75 \mathrm{US} \$ / \mathrm{KVAr}
\end{gathered}
$$

\subsection{Constraints} as follows:

The objective function in Eq. 20 is subjected to the power system equality and inequality constraints

\subsubsection{Equality constraints}

The equality constraints of the OPF including wind farms are the power flow equations as follows:

$$
\begin{aligned}
& P_{i}=P_{g i}+P_{w i}-P_{D i} \\
& Q_{i}=Q_{g i}+Q_{w i}-Q_{D i}
\end{aligned}
$$

where, $P_{i}$ and $Q_{i}$ are the bus active and reactive power injections can be expressed as:

$$
\begin{aligned}
& P_{i}=\mathrm{V}_{\mathrm{i}} \sum_{\mathrm{j} \in \mathrm{N}_{\mathrm{B}}} \mathrm{V}_{\mathrm{j}}\left(\mathrm{G}_{\mathrm{ij}} \cos \theta_{\mathrm{ij}}+\mathrm{B}_{\mathrm{ij}} \sin \theta_{\mathrm{ij}}\right) \mathrm{i} \in \mathrm{N}_{\mathrm{B}} \\
& Q_{i}=\mathrm{V}_{\mathrm{i}} \sum_{\mathrm{j} \in \mathrm{N}_{\mathrm{B}}} \mathrm{V}_{\mathrm{j}}\left(\mathrm{G}_{\mathrm{ij}} \sin \theta_{\mathrm{ij}}-\mathrm{B}_{\mathrm{ij}} \cos \theta_{\mathrm{ij}}\right) \mathrm{i} \in \mathrm{N}_{\mathrm{B}}
\end{aligned}
$$

\subsubsection{Inequality constraints}

These constraints are imposed on relevant variables to ensure that they satisfy physical limits of the devices. Active and reactive power supplied by each generator and generator voltages $V_{g}$ are limited to its 
maximum and minimum value. Where, generator active power $\left(P_{g i}\right)$, reactive power $\left(Q_{g i}\right)$ and voltage magnitudes $\left(V_{g i}\right)$.

$$
\begin{array}{ll}
P_{g i}^{\min } \leq E\left(P_{g i}\right) \leq P_{g i}^{\max }, & , i \in N_{G} \\
Q_{g i}^{\min } \leq E\left(Q_{g i}\right) \leq Q_{g i}^{\max }, & , i \in N_{G} \\
V_{g i}{ }^{\min } \leq E\left(V_{g i}\right) \leq V_{g i}{ }^{\max } & , i \in N_{G}
\end{array}
$$

Transformer taps setting, $\mathrm{t}_{\mathrm{k}}$, have min. and max. setting limits are stated as:

$$
t_{k}^{\min } \leq t_{k} \quad \leq t_{k}^{\max } \quad, i \in N_{T}
$$

Security limits: These constraints include the limits on load bus; voltage magnitudes $V_{D i}$, and branch flow $S_{l}$ limits they are stated as,

$$
\begin{array}{ll}
V_{D i}^{\min } \leq \mathrm{E}\left(V_{D i}\right) \leq V_{D i}^{\max } & , \mathrm{i} \in \mathrm{N}_{\mathrm{B}} \\
E\left(S_{l i}\right) \leq S_{l i}^{\max } & , \mathrm{i} \in \mathrm{N}_{\mathrm{L}}
\end{array}
$$

The setting parameters for FACTS controller are restricted by their limits as follow:

$$
\begin{array}{ll}
-0.8 X_{l} \leq X_{i T C S C} \leq 0.2 X_{l} & , i \in N_{T C S C} \\
-100 \mathrm{MVAr} \leq \mathrm{Q}_{\mathrm{iSVC}} \leq 100 \mathrm{MVAr} & , \mathrm{i} \in \mathrm{N}_{\mathrm{SVC}}
\end{array}
$$

The load factor $\lambda_{f}$ is controlled by its boundaries as:

$$
1 \leq \lambda_{f} \leq \lambda_{f}^{\max }
$$

\subsubsection{A constraint handling technique}

In order to efficiently handle the constraints, a constrained dominance concept is used in this paper [28]. The solution of the constraint $\mathcal{M}$ dominate the solution of the constraint $j$, if any of the following conditions is achieved:

- $\quad$ Both solutions $\mathcal{M}$ and $j$ are feasible solutions, and solution $\mathcal{M}$ dominates solution $j$.

- $\quad$ Solution $\mathcal{M}$ is feasible and solution $j$ is not.

- $\quad$ Both solutions $\mathcal{M}$ and $j$ are infeasible, but solution $\mathcal{M}$ has a lesser constraint violation.

In this study, the overall constraints violation can be considered as:

$$
J_{v}=W_{l} \cdot \sum_{i=1}^{N L} B L L_{i}+W_{v} \cdot \sum_{j=1}^{N B} V L_{j}
$$

$J_{v}$, is the factor indicating violation limits, BLL and $V L$ are associated to the line loading and the lines voltage level.

$$
\begin{aligned}
& B L L=\left\{\begin{array}{c}
1 ; \text { if } E\left(S_{p q}\right) \leq S_{p q} \max \\
e^{\left[\Upsilon \left(1-\frac{E\left(S_{p q}\right)}{\left.\left.S_{p q .} \text { max }\right)\right]}\right.\right.} ; \text { if } E\left(S_{p q}\right)>S_{p q} \text { max }
\end{array}\right. \\
& V L=\left\{\begin{array}{cc}
1 \quad ; \text { if } 1.1 \geq E\left(V L_{i}\right) \geq 0.95 \\
e^{\left[\mu\left(1-E\left(V L_{i}\right)\right]\right.} \quad ; \text { otherwise }
\end{array}\right.
\end{aligned}
$$

where $E\left(S_{l q}\right)$ and $S_{p q \text {. }}{ }^{\max }$ are the expected apparent power and thermal limit generated between buses p and q. $\Upsilon$ and $\mu$ are a small positive constant [7]. 


\section{SOLUTION APPROACH}

\subsection{Multi-objective particle swarm optimization}

The basic particle swarm optimization algorithm is developed exploiting social model simulations. The method is developed with inspiration from flocking of birds and schooling of fish. The PSO method was first designed to simulate behavior of birds searching for food in a bounded area. A single bird would find food through social cooperation with other birds in the flock, i.e., with its neighbors. The particle swarm optimization algorithm with dynamic neighborhood topology for every particle $(i=1,2, \ldots . ., N)$ can be described as [29].

$$
\begin{aligned}
& v^{i}(t+1)=\chi\left[v^{i}(t)+\varphi_{1}{ }^{i}\left(p^{i}(t)-x^{i}(t)\right)+\varphi_{2}{ }^{i}\left(g^{i}(t)-x^{i}(t)\right)\right] \\
& x^{i}(t+1)=v^{i}(t+1)
\end{aligned}
$$

where $x^{i}(t) \in \mathbb{R}^{n}$ is the position of $i$ th particle at time $t, p^{i}(t) \in \mathbb{R}^{n}$ is the best position achieved by the $i t h$ particle until time $t, g^{i}(t) \in \mathbb{R}^{n}$ is the best position achieved by ith particle and its neighbors until time $t$, $v^{i}(t) \in \mathbb{R}^{n}$ is the rate of position change (velocity) of the $i t h$ particle at time $t$, and $N$ is the number of particles in the swarm. The coefficients $\varphi_{1}{ }^{i}(t) \in\left[0, \varphi_{1}{ }^{-}\right]^{n}$ and $\varphi_{2}{ }^{i}(t) \in\left[0, \varphi_{2}{ }^{-}\right]^{n}$ are $n$-dimensional uniform vectors with random distribution referred to as social and cognitive learning coefficients, respectively. They determine the relative significance of social and cognitive components.

\subsection{Principles of MOPSO}

The MOPSO is an extension of original PSO given by [30], that is capable of handling many objective functions in one run.in MOPSO, position update and velocity update equations keep on same as in (47) and (48) in PSO. All the parameter declared are also same excluding the objective function. The comparison of PSO with other heuristic algorithms makes the obvious concept under consideration a Pareto set ranking procedure could be the straight way to develop the scheme to exploit the multi-objective optimization problems. The historical notation of optimum candidates obtained by a particle could be handled to keep non-dominated candidates generated in the previous iterations. By using of global attraction strategies under consideration a historical notation found non-dominated candidates would put through convergence characteristic into globally non-dominated solutions. The optimization problem is a multi-objective optimization which was considered as an effective method to discovery the optimal solution between different objectives. The detailed procedures for finding the best solutions by optimal Pareto set are introduced in Figure 2.

\subsection{Best compromise solution (BCS)}

As soon as the Pareto ideal group is got, it is commonsense to indicate single solution from all solutions that meet a few means to some expands. Fuzzy decision-making approach is intended to separate the BCS. For this optimization due to the inaccurate nature of the decision-making process involved, the ith objective function $f_{i}$ is denoted by a membership function $\mu_{i}$ specified as [31].

$$
\mu_{i}=\left\{\begin{array}{cl}
1 & F_{i} \leq F_{i, \min } \\
\frac{F_{i, \max }-F_{i}}{F_{i, \max }-F_{i, \min }} & F_{i, \min }<F_{i}<F_{i, \max } \\
0 & F_{i} \geq F_{i, \min }
\end{array}\right.
$$

where, $F_{i, \max }$ and $F_{i, \min }$ are the maximum and minimum value of the ith objective function amongst all non-dominated solution, respectively. For individually non-dominated solution $k$, the corresponding membership function $\mu^{k}$ is considered as:

$$
\mu^{k}=\frac{\sum_{i=1}^{N o b j} \mu_{i}^{k}}{\sum_{k=1}^{m} \sum_{i=1}^{N o b j} \mu_{i}^{k}}
$$

where $m$ is the number of non-dominated set, by using Fuzzy ranking method, the BCS from pareto front solutions can be selected and the best solution is the value with highest $\mu^{k}$. 


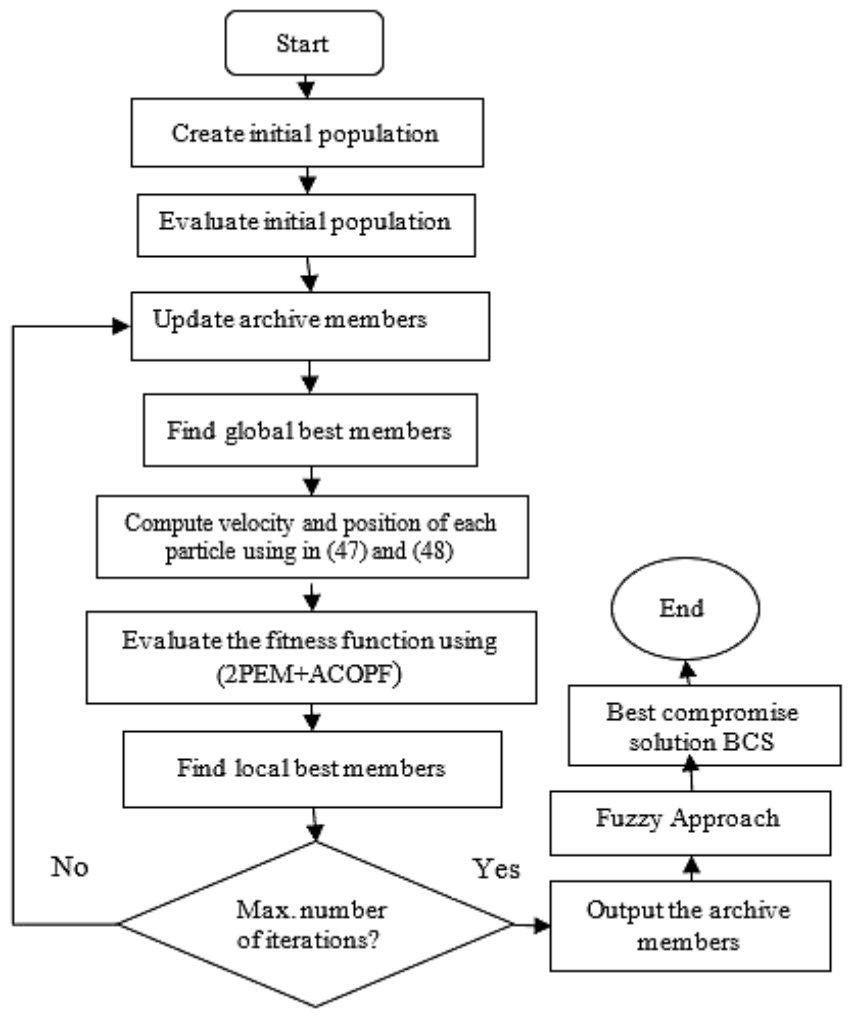

Figure 2. Flowchart of the proposed MOPSO algorithm

\subsection{Proposed solution for the FACTS allocation problem}

The optimal allocation of TCSC and SVC controller is expressed as hybrid continues-discrete MOP. The complete problem is planned as two levels. In the upper level the MOPSO look for the better solution through a number of feasible solutions, to get the location and rating of FACTS controller and the result of the first level is proceeded to second level. In the second level the (2PEM+1) is planned for the solution of the POPF problems, which are essential for the estimate of the fitness function for each particle of the PSO. The flowchart of the planned approach is appeared in Figure. 2. The step by step strategy of the proposed (MOPSO -2PEM) is explained below.

Step 1 : Enter the data of the power network, wind data and PSO parameters.

Step 2 : Set the location and rating of FACTS controller as a design variable.

Step 3 : Initialize the population as indicated by number of design parameters and population sizing.

Step $4:$ For each individual update the branch data for TCSC, bus data for SVC and running the probabilistic load flow using 2PEM method to estimate the value of objective functions for each particle.

Step 5 : Apply constraint handling technique.

Step 6 : Examine for the non-dominated results and store them in the external archive.

Step $7 \quad: \quad$ Update the velocity and position of population.

Step 8 : Compute the fitness value of objective functions for each individual using PEM.

Step $9:$ Examine for the non-dominated solutions and update the archive.

Step 10 : If the finished criterion is got, then go to next step else go to step 7.

Step 11 : Assess the expected objective function \&size and location of FACTS controller.

Step 12 : Apply Fuzzy approach.

Step 13 : Stop.

\section{SIMULATION RESULTS}

To excuse the efficiency of the planned method was tested on a modified IEEE 30-bus system, which consists of 30 buses and include six generators. Bus 1 is the slack bus. The network has 41 branches and 4 transformers. The network has total real and reactive load of 283.4MW and $126.2 \mathrm{MVAr}$, respectively. All data were taken as per-unit value and the base value was 100 MVA. The initial operating settings of 
the system are given by $[32,33]$.There are two variable speed wind farms connected at bus 14 and 19 as shown in Figure 3. Each farm has 24 identical wind turbines with the nominal capacities equal to $1.5 \mathrm{MW}$ at p. $\mathrm{f}=1$. The parameters of wind farms are given as follow: NEG Micon 1500/64 wind turbine, Scale

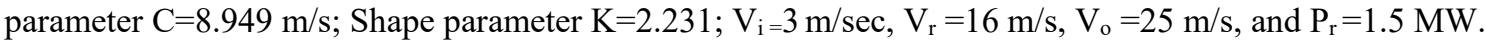

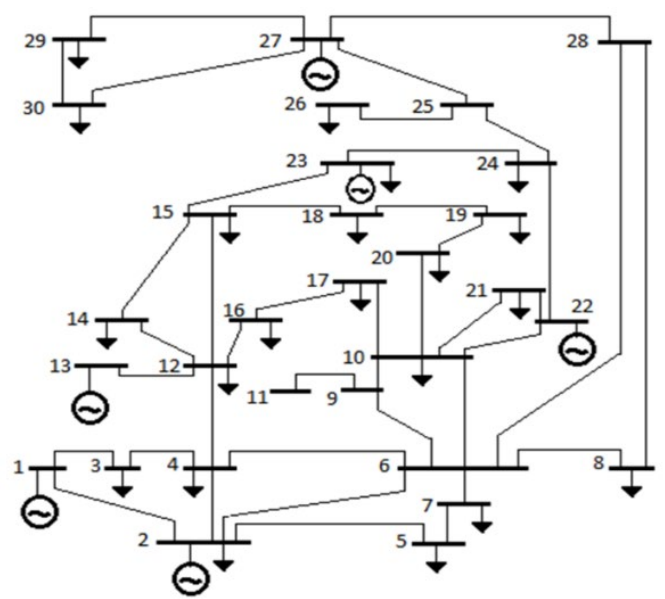

Figure 3. Modified IEEE 30-bus test system

Case 1: without FACTS controller

First, assume that FACTS controller has not been installed and the load factor $\lambda_{\mathrm{f}}$ is permitted in the certain of $[1,1.5]$. Additionally, the issue is managed as a MOP issue and expected power losses and system loadability are optimized are streamlined at the same time using MOPSO. The obtained results are shown in Figure 4, has archive member of the solutions got inside the security limits. Amongst the Pareto-ideal group, three of the solutions, which have the best system loadability, MSL, (F1), best real power losses (F2) and the (BCS) as illustrate from the Pareto front as shown in Figure 4. Also, the values of these solution are shown in Table 1. We can conclude from this result that when the uncertainty of wind farm output is considered, the system loadability is improved, the load factor $\lambda_{\mathrm{f}}$ is enhanced from 1.0 to 1.09 p.u, and the expectation of real power losses is increased.

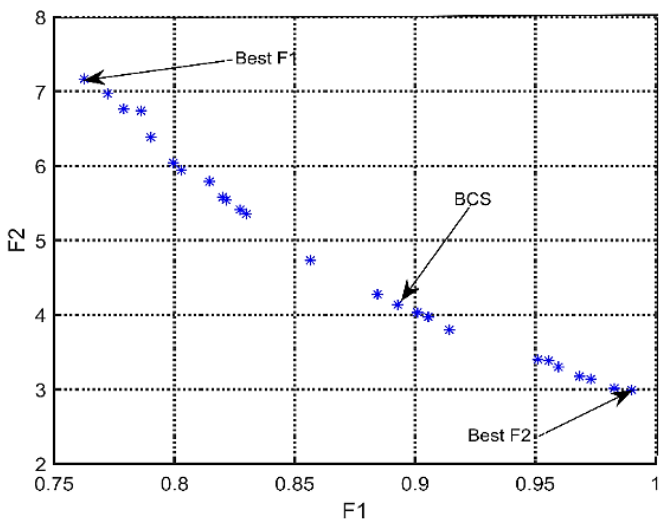

Figure 4. Archive member in two objective functions without FACTS devices

Table 1. Corresponding best objective function without FACTS devices

\begin{tabular}{ccc} 
Cases & $\mu$ [Losses] MW & $\lambda_{f}$ p.u \\
\hline Best F1 & 6.567 & 1.28 \\
Best F2 & 3.15 & 1.02 \\
BCS & 3.85 & 1.09 \\
Base case & 4.07 & 1.0 \\
\hline
\end{tabular}


Case 2. with FACTS controller

In that case, three different scenarios are considered the effect of FACTS controller on system loadability (F1), real power losses (F2) and the cost of installation FACTS controller (F3). For the first scenario 3 SVC are manage for seeking for optimal allocation simultaneously using MOPSO. next, one TCSC devices is considered. In the third, one TCSC and 3 SVC devices are considered. MOPSO is utilized to disband the problem and a group of non-dominated solutions has been got. These arrangements established the Pareto ideal set.

\section{Scenario 1: SVC only}

In the case of SVC, the variety of the Pareto optimal front through the tradeoff surface is shown in Figure 5 Clearly the arrangements are better apportioned on exchange off surface. Among the pareto ideal gathering, three arrangement which have the best of MSL, expected active power losses and establishment cost of FACTS controller. Table 2 shows the optimal location and rating of SVC as well as the MSL, expected active power loss, and installation cost of FACTS. This solution is selected based on the BCS as depicted from the Pareto front as shown in Figure 4. Furthermore, from all the non-dominated solutions in archive member the BCS gives the installation of SVC in buses 30, 14, 9 with the settings of 2.72, 2.81 and -1.57 MVAR. In addition, the obtained MSL is $115 \%$ and the minimum establishment cost for SVC is US\$ $0.958 \times 10^{6}$.

\section{Scenario 2: TCSC only}

In order to get it the finest rating and location of TCSC. Figure 6 illustrate the non-dominated solution for the optimal size and location of TCSC takes into account the maximization of MSL and the minimization of expectation of real power losses. The consequences for TCSC allocation have been given in Table 3. As shown in this table, the degree of compensation of TCSC is -0.74 p.u in line (10-20) gives MSL of $123 \%$ with the cost of installation is US $\$ 2.87 \times 10^{6}$.

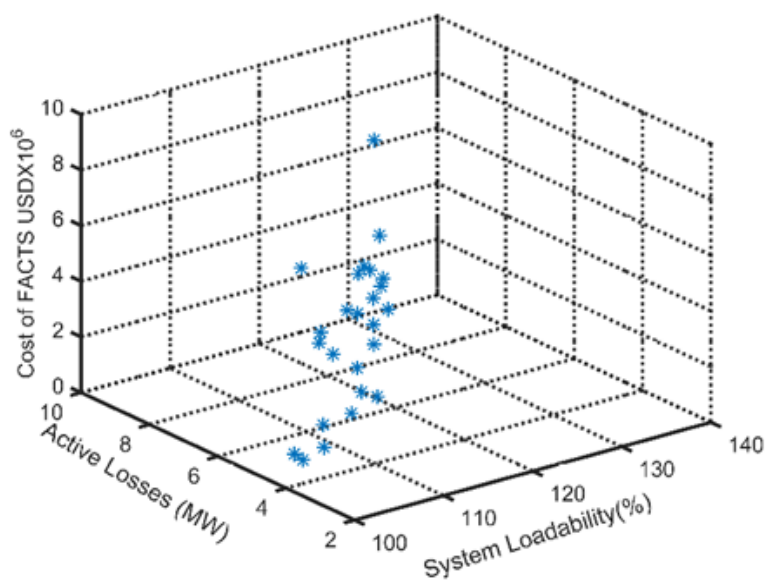

Figure 5. Archive member for location and rating of SVC

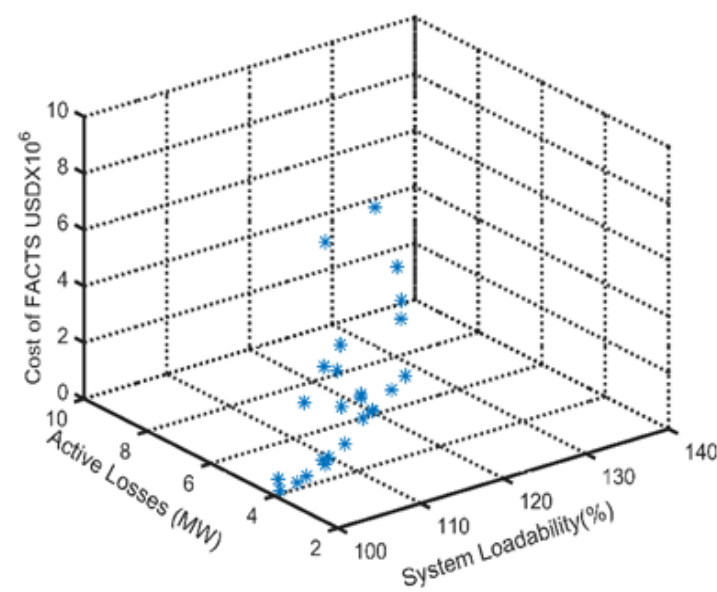

Figure 6. Archive member for location and rating of TCSC

Table 2. Optimal allocation of SVC for corresponding three objective function

\begin{tabular}{ccccccc}
\hline Case & FACTS Type & Bus No. & Rating & $\mu$ [Losses] MW & MSL\% & IC X106 USD \$ \\
\hline BCS & SVC & 30 & $2.72 \mathrm{MVAr}$ & 5.32 & 115 & 0.958 \\
& & 14 & $2.81 \mathrm{MVAr}$ & & & \\
& 9 & $-1.57 \mathrm{MVAr}$ & & & \\
\hline
\end{tabular}

Table 3. Optimal allocation of TCSC for corresponding three objective function

\begin{tabular}{ccccccc}
\hline Case & FACTS Type & Bus No. & Rating pu & $\mu$ [Losses] MW & MSL \% & IC X10 $0^{6}$ USD \$ \\
\hline BCS & TCSC & Line (10-20) & -0.74 & 6.25 & 123 & 2.87 \\
\hline
\end{tabular}




\section{Scenario 3: SVC and TCSC}

Figure 7 illustrate the non-dominated archive member solutions of best size and location of both SVC and TCSC. Actually, the obtained solutions are the best combinations of SVC and TCSC have been shown in Table 4. In this instance, MOPSO provides non-dominated solutions, where the installation of SVC of $(0.22,-5.05,4.412)$ MVAR size at the bus number (3-10-30) and the TCSC at the line (6-28) with a compensation degree of -0.79 pu level offer MSL of $119 \%$ and the establishment cost is US\$ $3.998 \times 10^{6}$.

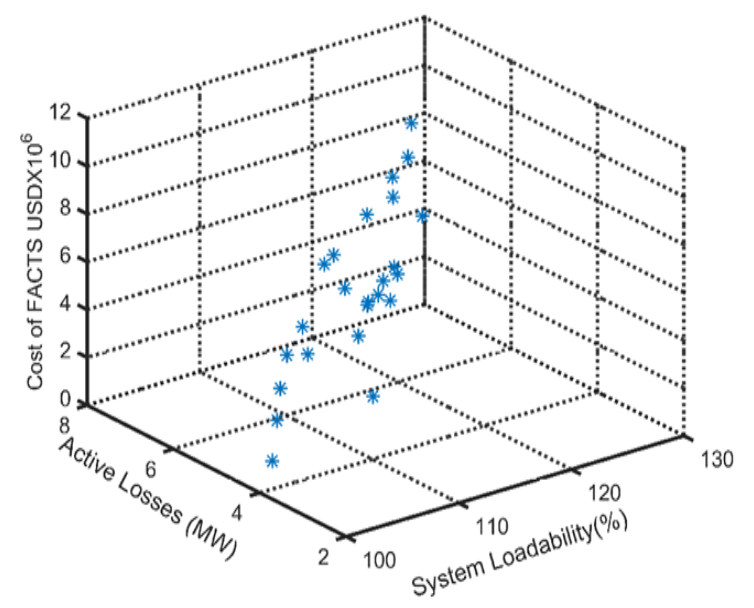

Figure 7. Archive member for location and size of (SVC-TCSC)

Table 4. Optimal allocation of TCSC and SVC for corresponding three objective function

\begin{tabular}{ccccccc}
\hline Case & FACTS Type & Bus No. & Ratingpu & $\mu$ [Losses]MW & MSL\% & IC X10 $10^{6}$ USD \$ \\
\hline BCS & TCSC & Line (10-20) & -0.74 & 6.25 & 123 & 2.87 \\
\hline
\end{tabular}

\section{CONCLUSION}

This study has introduced an efficient and basic methodology for choosing the ideal capacity and location of FACTS controller for networks with large scale of wind generation. The allocation of FACTS controller is presented are a multi-objective problem, the objective functions taken into consideration in the study were maximization of network loadability, minimization expectation network losses and the minimization the establishment cost of FACTS devices simultaneously. In this study, wind generation and load demand are considered as uncertainty are modeled using point estimate method while seeking for optimum allocation of FACTS controller. The effectiveness of the planned method is studied by applying it to the revised IEEE 30-bus system and solved using (MOPSO+2PEM). The outcomes show that suitable size and location of FACTS controller are extremely crucial by taking into account uncertainty.

\section{REFERENCES}

[1] G. W. E. Council, "Global Wind Report 2018," 2018.

[2] T. Ayodele, A. Jimoh, J. Munda, and J. Agee, "Challenges of grid integration of wind power on power system grid integrity: A review," International Journal of Renewable Energy Research, vol. 2, no. 4, pp. 619-626, 2012.

[3] P. Kotsampopoulos, P. Georgilakis, D. T. Lagos, V. Kleftakis, and N. Hatziargyriou, "FACTS Providing Grid Services: Applications and Testing," Energies, vol. 12, no. 13, pp. 1-23, 2019.

[4] B. Singh, "Introduction to FACTS controllers in wind power farms: A technological review," International Journal of Renewable Energy Research (IJRER), vol. 2, no. 2, pp. 166-212, 2012.

[5] S. Pati and R. Dahiya, "Optimal location of TCSC in a large system to optimize load Flow: A sensitivity based approach," in 2016 IEEE 6th International Conference on Power Systems (ICPS) IEEE, pp. 1-5, 2016.

[6] A. Rezaeejordehi and J. Jasni, "A comprehensive review on methods for solving FACTS optimization problem in power systems," International Review of Electrical Engineering, vol. 6, no. 4, pp. 1916-1926, 2011.

[7] M. Saravanan, S. M. R. Slochanal, P. Venkatesh, and J. P. S. Abraham, "Application of particle swarm optimization technique for optimal location of FACTS devices considering cost of installation and system loadability," Electric power systems research, vol. 77, no. 3-4, pp. 276-283, 2007.

[8] S. S. Reddy, "Determination of optimal location and size of static VAR compensator in a hybrid wind and solar power system," International Journal of Applied Engineering Research, vol. 11, no. 23, pp. 11494-11500, 2016. 
[9] N. Cherkaoui, A. Belfqih, F. El Mariami, J. Boukherouaa, and A. Berdai, "Optimal location and reactive power injection of wind farms and SVC's units using voltage indices and PSO," International Journal of Electrical and Computer Engineering (IJECE), vol. 6, no. 6, pp. 2581-2588, 2019.

[10] M. Aghaebrahimi, R. K. Golkhandan, and S. Ahmadnia, "Localization and sizing of FACTS devices for optimal power flow in a system consisting wind power using HBMO," in 2016 18th Mediterranean Electrotechnical Conference (MELECON), pp. 1-7, 2016.

[11] S. S. Reddy, "Optimal Placement of FACTS Controllers for Congestion Management in the Deregulated Power System," Transmission and Distribution Conference and Exposition, IEEE PES, 2010.

[12] K. Bavithra, S. C. Raja, and P. Venkatesh, "Optimal setting of FACTS devices using particle swarm optimization for ATC enhancement in deregulated power system," IFAC-PapersOnLine, vol. 49, no. 1, pp. 450-455, 2016.

[13] M. J. Morshed, J. B. Hmida, and A. Fekih, "A probabilistic multi-objective approach for power flow optimization in hybrid wind-PV-PEV systems," Applied energy, vol. 211, pp. 1136-1149, 2018.

[14] S. M. Mohseni-Bonab, A. Rabiee, B. Mohammadi-Ivatloo, S. Jalilzadeh, and S. Nojavan, "A two-point estimate method for uncertainty modeling in multi-objective optimal reactive power dispatch problem," International Journal of Electrical Power \& Energy Systems, vol. 75, pp. 194-204, 2016.

[15] M. Aien, M. Rashidinejad, and M. F. Firuz-Abad, "Probabilistic optimal power flow in correlated hybrid wind-PV power systems: A review and a new approach," Renewable and Sustainable Energy Reviews, vol. 41, pp. 1437-1446, 2015.

[16] S. O. Faried, R. Billinton, and S. Aboreshaid, "Probabilistic technique for sizing FACTS devices for steady-state voltage profile enhancement," IET generation, transmission \& distribution, vol. 3, no. 4, pp. 385-392, 2009.

[17] S. Galloway, I. Elders, G. Burt, and B. Sookananta, "Optimal flexible alternative current transmission system device allocation under system fluctuations due to demand and renewable generation," IET generation, transmission \& distribution, vol. 4, no. 6, pp. 725-735, 2010.

[18] S. Galvani, M. T. Hagh, M. B. B. Sharifian, and B. Mohammadi-Ivatloo, "Multiobjective Predictability-Based Optimal Placement and Parameters Setting of UPFC in Wind Power Included Power Systems," IEEE Transactions on Industrial Informatics, vol. 15, no. 2, pp. 878-888, 2018.

[19] R. Billinton and D. Huang, "Effects of load forecast uncertainty on bulk electric system reliability evaluation," IEEE Transactions on Power Systems, vol. 23, no. 2, pp. 418-425, 2008.

[20] R. Bo and F. Li, "Probabilistic LMP forecasting considering load uncertainty," IEEE Transactions on Power Systems, vol. 24, no. 3, pp. 1279-1289, 2009.

[21] R. Karki, P. Hu, and R. Billinton, "A simplified wind power generation model for reliability evaluation," IEEE transactions on Energy conversion, vol. 21, no. 2, pp. 533-540, 2006.

[22] C. Schauder et al., "Development of a/spl plusmn/100 MVAr static condenser for voltage control of transmission systems," IEEE Transactions on Power Delivery, vol. 10, no. 3, pp. 1486-1496, 1995.

[23] S. Gerbex, R. Cherkaoui, and A. J. Germond, "Optimal location of multi-type FACTS devices in a power system by means of genetic algorithms," IEEE transactions on power systems, vol. 16, no. 3, pp. 537-544, 2001.

[24] G. Verbic and C. A. Canizares, "Probabilistic optimal power flow in electricity markets based on a two-point estimate method," IEEE transactions on Power Systems, vol. 21, no. 4, pp. 1883-1893, 2006.

[25] M. Aien, M. Fotuhi-Firuzabad, and M. Rashidinejad, "Probabilistic optimal power flow in correlated hybrid wind-photovoltaic power systems," IEEE Transactions on Smart Grid, vol. 5, no. 1, pp. 130-138, 2014.

[26] A. H. Dias and J. A. De Vasconcelos, "Multiobjective genetic algorithms applied to solve optimization problems," IEEE Transactions on Magnetics, vol. 38, no. 2, pp. 1133-1136, 2002.

[27] H. Jain and K. Deb, "An evolutionary many-objective optimization algorithm using reference-point based nondominated sorting approach, part II: handling constraints and extending to an adaptive approach," IEEE Transactions on Evolutionary Computation, vol. 18, no. 4, pp. 602-622, 2013.

[28] K. Deb, A. Pratap, S. Agarwal, and T. Meyarivan, "A fast and elitist multiobjective genetic algorithm: NSGA-II," IEEE transactions on evolutionary computation, vol. 6, no. 2, pp. 182-197, 2002.

[29] C. C. Coello and M. S. Lechuga, "MOPSO: A proposal for multiple objective particle swarm optimization," in Proceedings of the 2002 Congress on Evolutionary Computation. CEC'02 (Cat. No. 02TH8600), vol. 2, IEEE, pp. 1051-1056, 2002.

[30] L. Li, W. Wang, and X. Xu, "Multi-objective particle swarm optimization based on global margin ranking," Information Sciences, vol. 375, pp. 30-47, 2017.

[31] A. L. Ara, A. Kazemi, and S. N. Niaki, "Multiobjective optimal location of FACTS shunt-series controllers for power system operation planning," IEEE Transactions on Power Delivery, vol. 27, no. 2, pp. 481-490, 2011.

[32] O. Alsac and B. Stott, "Optimal load flow with steady-state security," IEEE transactions on power apparatus and systems, vol. PAS-93, no. 3, pp. 745-751, 1974.

[33] R. D. Zimmerman, C. E. Murillo-Sánchez, and R. J. Thomas, "MATPOWER: Steady-state operations, planning, and analysis tools for power systems research and education," IEEE Transactions on power systems, vol. 26, no. 1, pp. 12-19, 2010. 